\title{
Predictores de resultado neonatal adverso en colestasia intrahepática del embarazo (1)
}

\author{
Kawakita T, Parikh LI, Ramsey PS, Huang Ch Ch, Zeymo A, Fernandez M, Smith S, Iqbal \\ SN. Predictors of adverse neonatal outcomes in intrahepatic cholestasis of pregnancy. \\ Am J Obstet Gynecol 2015. pii: S0002-9378(15)00604-3. doi: 10.1016/j.ajog.2015.06.021.
}

Análisis crítico: Carolina Martinovic Titiro, Jorge A. Carvajal, PhD.

Unidad de Medicina Materno-Fetal, División de Obstetricia y Ginecología, Escuela de Medicina, Pontificia Universidad Católica de Chile.

\section{RESUMEN (1)}

Objetivo: Determinar predictores de resultado adverso neonatal en mujeres con colestasia intrahepática del embarazo. Método: Estudio de una cohorte retrospectiva multicéntrica de todas las mujeres diagnosticadas con colestasia intrahepática del embarazo a través de 5 hospitales entre enero del 2009 y diciembre del 2014. Complicaciones obstétricas y neonatales fueron evaluadas de acuerdo al niveles de acidos biliares totales. Modelos de regresión logística multivariable fueron desarrollados para evaluar predictores de resultado neonatal compuesto (admisión a unidad de cuidados intensivos neonatales, hipoglicemia, hiperbilirrubinemia, síndrome de distrés respiratorio, taquipnea transitoria del recién nacido, uso de ventilación mecánica, aporte de oxigeno por cánula nasal y feto muerto in útero). Predictores, incluyendo nivel de ácidos biliares totales, nivel de transaminasas hepáticas, edad gestacional al diagnóstico, enfermedad hepática previa y uso de acido ursodeoxicólico fueron evaluados. Resultados: De 203 mujeres con colestasia intrahepática del embarazo, 152 pacientes tuvieron niveles de ácidos biliares totales entre $10-39,9 \mu \mathrm{mol} / \mathrm{L}$; 55 tuvieron niveles de ácidos biliares totales 40 - 99,9 $\mu \mathrm{mol} / \mathrm{L}$ y 26 tuvieron niveles de ácidos biliares totales $\geq 100$ $\mu \mathrm{mol} / \mathrm{L}$. No hubo diferencias en cuanto a edad materna, raza, índice de masa corporal pre embarazo de acuerdo a niveles de ácidos biliares totales. Aumento de los niveles de ácidos biliares totales fue asociado con aumento de las transaminasas y nivel de bilirrubina total $(p<0,05)$. Niveles de ácidos biliares totales $\geq 100 \mu \mathrm{mol} / \mathrm{L}$ se asociaron con aumento del riesgo de mortinato $(p<0,01)$. Aumento de los niveles de ácidos biliares fue asociado con edad gestacional temprana al diagnóstico $(p<0,01)$ y uso de ácido ursodeoxicólico $(p=0,02)$. Después de ajustar por variables confundentes ningún predictor fue asociado con morbilidad neonatal compuesta. Niveles de ácidos biliares totales 40 - 99,9 $\mu \mathrm{mol} / \mathrm{L}$ $y \geq 100 \mu \mathrm{mol} / \mathrm{L}$ se asociaron con aumento del riesgo de meconio en liquido amniótico (OR ajustado 3,55 ; [95\% IC 1,45-8,68] y OR ajustado 4,55 [95\% IC 1,47-14,08], respectivamente). Conclusión: En mujeres con colestasia intrahepatica del embarazo niveles de ácidos biliares totales $\geq 100 \mu \mathrm{mol} / \mathrm{L}$ se asoció a aumento del riesgo de mortinato. Niveles de ácidos biliares totales $\geq 40 \mu \mathrm{mol} / \mathrm{L}$ fue asociado con aumento del riesgo de tinte meconial en el líquido amniótico.

\section{ANÁLISIS DE LA INVESTIGACIÓN}

\section{A. Relevancia clínica de la investigación}

La colestasia intrahepática del embarazo es una patología que se produce en el segundo y tercer trimestre del embarazo, se caracteriza por prurito y elevación de la concentración sérica de ácidos biliares (2). La fisiopatología de la enfermedad es desconocida aunque factores genéticos ambientales y hormonales influyen en la expresión de la enfermedad. La colestasia intrahepática del embarazo, en general, pocas veces presenta 
complicaciones maternas, sin embargo se asocia a complicaciones fetales como son parto prematuro entre el 6 al $60 \%$, ya sea espontáneo o iatrogénico, y muerte fetal in útero en el 1 a $1,2 \%$ sobre las 37 semanas, momento en el que ocurre el $90 \%$ de las muertes (2-3). Se cree que la causa de la muerte fetal sería el desarrollo de arritmias fetales o vasoespasmo del lecho placentario, ambos eventos que no se han logrado predecir con ninguna técnica de vigilancia antenatal. Por ello, se hace muy relevante el estudio de algún factor que pudiera predecir resultado adverso perinatal y así poder definir el momento más apropiado y seguro para la interrupción del embarazo, balanceando el riesgo de mortalidad y de prematurez. Nuestra norma en la Pontificia Universidad Católica de Chile es monitorización semanal de las mujeres con CIE mediante Registro de la Frecuencia Cardiaca Basal No Estresante (RBNE), e indicar la interrupción del embarazo a las 38 semanas en CIE anictérica y a las 36 semanas si se presenta ictericia (4). En la literatura internacional, si bien no existe un consenso, se recomienda la interrupción del embarazo entre las 35 y las 37 semanas de gestación, ya que es ese el momento en que el riesgo por prematurez se disminuye versus el riesgo de resultado perinatal adverso asociado al manejo expectante (3-5).

\section{B. El estudio (1)}

Diseño: Cohorte retrospectiva multicéntrica de 5 hospitales: MedStar Washington Hospital Center (Washington, DC), MedStar Georgetown University Hospital (Washington, DC), Medstar Franklin Square Medical Center (Baltimore, MD), Medstar Harbor Hospital (Baltimore, MD), and Virginia Hospital Center (Arlington, VA) que incluyó a todas las embarazadas diagnosticadas con colestasia intrahepática del embarazo (CIE) entre enero del 2009 y diciembre del 2014. Las complicaciones neonatales y obstétricas fueron evaluadas de acuerdo al nivel total de acidos biliares. Se desarrollaron modelos de regresión logística multivariable para evaluar predictores de resultado adverso neonatal compuesto. Resultado principal: se evaluó el resultado adversos neonatal compuesto, considerando como tal: admisión a unidad de cuidados intensivos neonatales, hipoglicemia, hiperbilirrubinemia, síndrome de distrés respiratorio, taquipnea transitoria del recién nacido, necesidad de ventilación mecánica, necesidad de aporte de oxígeno por cánula nasal y mortalidad fetal in útero. Predictores: Los predictores evaluados fueron los niveles de ácidos biliares totales, nivel de transaminasas, edad gestacional al diagnóstico, enfermedad hepática previa y uso de ácido ursodeoxicólico. Participantes: 233 pacientes con diagnóstico clínico de colestasia intrahepatica del embarazo y valor de acidos biliares totales $>10 \mu \mathrm{mol} / \mathrm{L}$. Se excluyó a las pacientes con gestaciones múltiples, anomalías congénitas, alteraciones cromosómicas y valor de ácidos biliares totales $<10 \mu \mathrm{mol} / \mathrm{L}$. Resultados: Las pacientes fueron divididas en grupo según el nivel plasmático de ácidos biliares totales: Grupo 1 ácidos biliares totales 10 - 39,9 $\mu \mathrm{mol} / \mathrm{L}$ (152 pacientes); Grupo 2 ácidos biliares totales 40 - 99,9 $\mu \mathrm{mol} / \mathrm{L}$ (55 pacientes); Grupo 3 ácidos biliares totales $\geq 100 \mu \mathrm{mol} / \mathrm{L}$ (26 pacientes). El aumento de los niveles de ácidos biliares se asoció con aumento de las transaminasas y nivel total de bilirrubina $(p<0,05)$. Mujeres con niveles de ácidos biliares totales 40 - 99,9 $\mu \mathrm{mol} / \mathrm{L}$ $y \geq 100 \mu \mathrm{mol} / \mathrm{L}$ tuvieron más parto prematuro (<37 semanas) iatrogénico $(p<0,01)$. Niveles de acidos biliares totales $\geq 100 \mu \mathrm{mol} / \mathrm{L}$ se asociaron con el aumento del riesgo de mortinato $(p<0,01)$. Los 4 casos de mortinato se presentaron en el grupo de niveles de ácidos biliares totales $\geq 100 \mu \mathrm{mol} / \mathrm{L}$. El aumento de los niveles de ácidos biliares 40 - 99,9 $\mu \mathrm{mol} / \mathrm{L}$ y $\geq 100 \mu \mathrm{mol} / \mathrm{L}$ fue asociado con edad gestacional temprana al diagnóstico $(p<0,01)$ y uso de ácido ursodeoxicólico $(\mathrm{p}=0,02)$. Después de ajustar por variables confundentes ningún predictor fue asociado con morbilidad neonatal compuesta. Los niveles de ácidos biliares totales 40 - 99,9 $\mu \mathrm{mol} / \mathrm{L}$ $y \geq 100 \mu \mathrm{mol} / \mathrm{L}$ se asociaron con el aumento del riesgo de meconio en líquido amniótico OR 3,55; (95\%IC $1,45-8,68)$ y OR 4,55 (95\%IC $1,47-14,08)$, respectivamente.

\section{Análisis crítico}

Validez interna: El diseño del estudio cohorte retrospectiva es apropiado para poder establecer un factor predictor o una asociación estadística. El hecho de que sea un estudio multicéntrico permite tener heterogeneidad en la población de estudio. No existen diferencias estadísticamente significativas en cuanto a edad materna, raza, paridad, índice de masa corporal, síndrome hipertensivo del embarazo, diabetes, historia de enfermedad hepática previa o inducción del trabajo de parto en los grupos seleccionados por nivel de ácidos biliares totales. Sin embargo existe pérdida de información: se excluyeron 131 pacientes por falta de los niveles de ácidos biliares totales lo que podría generar sesgo de información en los resultados. No se compararon los resultados adversos obtenidos con los resultados adversos de la población estándar de referencia para poder evaluar cual es la prevalencia de ellos, y si la CIE se asocia a mayor frecuencia de resultado perinatal adverso en la población. Validez externa: El estudio no es aplicable a nuestra población ya que las diferencias étnicas con nuestra población determinan una prevalencia distinta de la enfermedad. En Estados Unidos se han reportado incidencias de $0,32 \%$ y en el estudio se encontró una prevalencia de $0,58 \%$ mientras que la población chilena se presenta en 1 a 
$2 \%$ de los embarazos. Además los ácidos biliares, predictor usado para estratificar las pacientes con los resultados adversos perinatales, no se miden en forma habitual en nuestro medio como parte del manejo de esta patología. Conclusión: Estudio con buen diseño para establecer asociación estadística, sin embargo con pérdidas de información por error de registro, que pone en riesgo la veracidad de los resultados. Al ser ajustados los resultados por variables confundentes, ningún predictor fue asociado con morbilidad neonatal compuesta, es decir este estudio no permite establecer un factor predictor de resultado adverso perinatal en pacientes con CIE. La información de este artículo no es útil para cambiar nuestra práctica clínica habitual, pero alerta sobre la necesidad de medir los niveles de ácidos biliares para evaluar si niveles muy altos se asocia a mayor riesgo de óbito fetal como sugiere este estudio.

\section{REFERENCIAS}

1. Kawakita T, Parikh LI, Ramsey PS, Huang Ch Ch, Zeymo A, Fernandez M, Smith S, lqbal SN . Predictors of adverse neonatal outcomes in intrahepatic cholestasis of pregnancy. Am J Obstet Gynecol 2015. pii: S0002-9378(15)00604-3. doi: 10.1016/j. ajog.2015.06.021. [Epub ahead of print].
2. Bacq $Y$. Liver diseases unique to pregnancy: $A$ 2010 update. Clin Res Hepatol Gastroenterol 2011;35(3):182-93.

3. Puljic A, Kim E, Page J, Esakoff T, Shaffer B, LaCoursiere DY, Caughey AB. The risk of infant and fetal death by each additional week of expectant management in intrahepatic cholestasis of pregnancy by gestational age. Am J Obstet Gynecol 2015;212(5):667. e1-5.

4. Rioseco A, Ivankovic MB, Manzur A, Hamed F, Kato SR, Parer JT, Germain AM. Intrahepatic cholestasis of pregnancy: a retrospective case-control study of perinatal outcome. Am J Obstet Gynecol 1994;170(3):890-5.

5. Brouwers L, Koster MP, Page-Christiaens GC, Kemperman $\mathrm{H}$, Boon J, Evers IM, Bogte A, Oudijk MA. Intrahepatic cholestasis of pregnancy: maternal and fetal outcomes associated with elevated bile acid levels. Am J Obstet Gynecol 2015;212(1):100.e1-7. 